\section{UN EFFORT D’ORGANISATION NATIONALE}

(Suite)

Le nombre des comités constitués est à l'heure actuelle de trente-trois : mais la liste n'en est pas close : et il en sera constitué de nouveaux, au moment opportun, pour d'autres wuvres intóressantes.

11 ine paraît intércssant d'en prósenter ci-après la liste en laccompagnant de quelques brèves indications sur l'objet ul l'étal d'avancement de leurs travaux :

\section{a) Voirie routièrt \\ b) Navigation intérieure}

Travaux d'amélioration des voies navigables. - Ce comité soccupe de lamélioration à apporter aux voies navigables existanleo autres que celles pour lesquelles des comités spédiaus ont été institués.

Il est chargé d'examnier, d'une part les améliorations d'ordre technique que ces voies peuvent exiger : augmenhation du mouillage, accroissement des dimensions de la cuvelle, des écluses, des ponts ; perfectionnement des barrag'es el réservoirs, etc. ; d'autre part, les moyens propres à améliorer l'exploitation : organisation de services de trac lion, accroissement des moyens d'alimentation ; établissemenl de ports et de raccordements avec les voies ferrées outillage de chargement et de déchargement dans les ports constitution d'un outillage perfectionné pour les travaux d'entretien et de réparations en vue notamment de supprimer ou de diminuer les chòniages, etc.

Le comité a procédé à une enquète générale dans les ser vices de navigation pour recueillir leurs propositions à ces divers points de vue. Les documents sont aujound'hui à peu près complètement recueillis.

Un soccupe de les coordonner pour présenter en comité le travail d'ensemble sur lequel il aura à délibérer.

Canaux du Nord, du Nord-Est et voies navigables du Nord el du Nord-Est. - Ce comité s'est occupé d'abord du canal du Nord, dont la reconstruction présente un caractère particulier d'urgence : au cours des enquêtes qui ont précédé la déclaration d'utilité publique, la question s'était posée de savoir si le canal serait construit pour les bateaux du type péniche ou pour les chalands de 500 à 600 tonnes ; la première solution avait prévalu contre l'avis de la plupart des usagers des voies navigables ; cependant les écluses ont été construites avec des longueurs permettant l'accès de bateaux de grande longueur et de fort tonnage sous la largeur de cinq mètres ; mais ce type de bateau auratt l'inconvénient de présenter des difficultés de construction conduisant à un prix de revient élevé et, d'autre part, la manœuvre en rivière de chalands de grande longueur sous faible largeur et faible mouillage, pourrait offrir de sérieux dangers. Le canal du Vord étant partiellement détruit, le comité s'est demandé sill ne conviendrait pas d'étudier à nouveau la question. l'élargissement de la ouvelte, la reconstruction d'écluses d'un type nouveau, ne présenteraient pas de difficultés.

A la suite de cette délibération, l’ingénieur en chef du service a été chargé d'examiner l'affaire ; le canal mis à grande section, constituerait d'abord une vole tout à fait perfectionnée pour l'adduction à Paris des charbons de la région du Nord, et, ensuite, formerait l'artère principale d'une grande ligne navigable de Paris à Dunkerque.

Le comité sera très prochainement réuni :

$\mathrm{r}^{*}$ Pour se prononcer sur cette question ; $2^{\circ}$ Pour entendre le président de l'association générale de la navigation intérieure ;

$3^{\circ}$ Pour examiner une demande, présentée par le Conseil municipal de Vouziers, tendant à ce que l'embranchement du canal des Ardennes soil prolongé jusqu'au canal de la Marne au Rhin.

Aménagement de la Seine entre Montereau et la mer. Le comité a été saisi d'un programme d'avant-projet comportant l'approfondissement de la Seine à $4 \mathrm{~m}$. 5o, entre Bougival et Rouen; il en a partagé l'examen entre ses menbres : l'étude technique na donné lieu à aucune critique ; on a dû réserver cependant la question à l'estimation des dépenses évaluées à 450 millions qui, à raison surtout des circonstances, ne repose et ne pouvait reposer que sur des bases incertaines.

L'étude économique et l'étude financière se poursuivent, Néanmoins, pour répondre à une denande du ministre, le comité a formulé, à l'unanimité, un avis où il indique les travaux quil est possible d'engager immédiatement, en ré:ervant l'avenir.

Une réunion est prévue pour entendre le président de l'association générale de la navigation intérieure, discuter les rapports sur la question économique et financière, et examincr une demande présentée par le prófet de la Seine en vue de la concession à la ville des ports de Paris et du déparlement de la Seine.

Loire, de Nantes à Bniare. - Par suite de circonstances particulières, la constitution de ce comité est de date très récente. Son président s'occupe de réunir les documents relatifs aux études antérieures qui, d'ailleurs, sont nombreuses et peuvent être considérées comme à peu près complètes. Le comilé paraît devoir donc être en mesure de formuler son avis dans un délai asse $z$ bref.

Canal de la Garonne à la·Loire. - Après un examen général de la quesion, une revision des études antérieures et un premier échange de vues, le comité a estimé que le premier. travail à entreprendre était de rechercher si les raisons subsistaient toujours qui ont fait jusqu'à présent écarter l'exécution d'un canal reliant la Loire à la Garonne, évalué, il y a dix ans, ì la somme de 300 millions environ sujvant la soluIıon la moins coûteuse ou si, alı contraire, les circonstances nées de la guerre et la possibilité d'associer la navigation à l'utilisation des forces hydrauliques des rivières de la région, ne sont pas de nature a donner aujound hui à cette entreprise un caraclère d'intérêt national.

Le comilé s'occupe d'organiser, avec le concours de l'oflice des transports du centre-ouest, les enquêtes qui permetIront d'élucider cette question.

Canal de Berry, canal de Moulins à Sancoins. - La queslion de la mise au gabarit nomal du canal de Berry et celle de la constructoon d'un canal de Moulins à Sancoins sont intimement liées, à de nombreux points de vue, à celle de l'exécution d'un canal de la Loire à la Garonne, et d'un canal latéral à l'Allier.

Les études sont poursuivies parallelement, et comportent, d'ailleurs, sou certains rapports, les mêmes recherches.

Canal de la loire au Rhône. - Le comité, après examen des citudes techniques et économiques antérieures, a désigné doux des ses membres pour réunir les documents propres à justifier l'utilité du canal dans les différentes hypothèses susceptibles d'être envisagées, savoir

Canal de Roanne à Rotarieux.

Canal de Saint-Etienne à Givors.

Canal de Roanne à Saint-Etienne et à Givors. 
Cimal de Roanne à Saint-Etienne ot à Givors prolongé jusqu’à Lyom.

Des queshronmaires ont élé cnvoyés aux intéressés.

Lassociaticn générale de navigalion intérieure sera entendue lor: de la prochaine rónion du comilé.

Voies navgables du Sind-()uest (canal latéral à la Garomne, canal du Midi, (iurumne, Dojdogne, Isle, Lol, Basse, Tarn...).

(ie conilé vienl reulemen̂t l'ètre constitué : on a dû altendre pour lorgani-er la production du rapport dune mission spécrale presorte par le ministre pour servir de base i ses latraul.

Ge rappont vienl dène prénenté le comité va immédiaterient se molles a loeuve.

Aménagement du Rhòne au point de vue de la navigation, des forces metricen el de l'agriculture - - A l'époque où le comilé du Rhòn a dx conslilué, sa tàche était limitée à l'cxamon des solutions qui ćlaient depuis longtemps à l'étude pour lubilisation des forees hydrauliques du Rhòne dans la parlie supérienre de suirse.

Sra mission n’a pas lardé à s'étendre à l'étude de l'aménagement inléghl du llouve, entre la suise et la mer, au triple point de vur de la foree molrice, de la navigation el des améholations agricoles.

Pour la solution do cet important et dufficile problème,

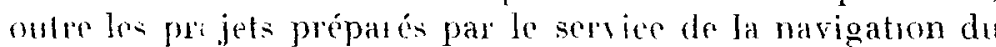
Shone, te comitć a été saini de ders éludes dont il a entendu les auteurs en leurs explications.

D'autres projels sont annoncés qu'il examinera le moment wnu. les rapponlems désignés procèdent it lietude de ces projets.

Des rapporl onl élé déposés au sujet de laménagement hydromedifque el de la question des irrigations dans la vallée du Rhone à laval de Lyon.; ils scront prochainement discutes par le comulé.

la création d'un port à Lyon a fait l'objet d'une délibéralion el le comité a désigné deux de sos membres pour établir in rappost ì ce sujel.

L.élude óconomique est en cours.

In projed de convention internationale entre la France at la Susse a ché rédigé pour préciser les point. sur lesquels l'entente est mócessaire entre les deux pays en vuc d'assurer la comlimuité de la navigation. Les pourparlers au sujel de celle convention ont déjà tété engagés entre des représentants lochniques des deux pays.

Enlin, le comilé s'esl occupé de préparer le cadre du projet do loi suivant lequel pourrait itre engagé l'aménagement iolégral du Rhóne, en s’inspirant de la forme adoptée pour les dernicrs progranmes de travaux du Havre et de Marseille

D’autre part, à un point de vute moins général, a été longuement cxaminée la question du choix à faire entre le. cmiplacements concurrents, Génısial ou Malpertuis et Bellegarde, pour la construction des grands barrages à établir dans la partic supérieure du Haut-Rhòne. Le comité a reconnu nécessaire, pour se déterminer, de faire procéder à des sondages qui sont actucllement en cours.

Les travalx du comilé ont comporté de très nombreuses auditions, il a culendu, notamment, les demandeurs en conces-ion des chutes de Génissiat et de Malpertuis-Bellegarde, les géolngues qui ont étudié les sites des barrages. cortains représonlantis des corps élus des région intéressées. des délégués du conseil municipal de Paris, des représentants des sociétés suieses qui poursuivent la jonction du Rhône au Rhin à travers Je territoire helvétique, des délégúés de l'asso- ciation générale de navigation intérieure, les auteurs des divers projebs dont il a été saisi, etc.

la. Làche du comité, en rapport avec l'muportance el la difliculté exceptionnelle de l'ceuvie à réaliser', est considérable. Tout donne lieu d'espérer qu'elle ne lardera pas à ètre inenće à bonne lin.

Canal du Rhòne au Rhrn. - Lamélioration de la voic navigable, joiguant te Rhône au Rhin ì travers le territoine français, présente, à raison du retour de l'Alsace à la France, une urgence el un inlérèt de promier crdre.

Ic comilé d'études a déjà formulé des conclusions tendant à ce que le canal existant soit mis immédiatentent aux frais (le l'Etat, au gabrait de la loi du 5 aoùt 1879 , afin dy permeltre la crrculation de la péniche de 300 lonnes. Cette propostlion a élé soumusc à la $2^{\circ}$ section du conseil supéricur qui la appryée.

Le comité n'en poursult pas moins une élude en vue de la modificalion ultéricure de cette voie navigable, de manière a la meltre en étal de luver passage aux grands baleau du Rhòne el du Rhin.

\section{c) Forces hydrauliques.}

Comité el sous-combés des forces hydrauliques. - Ce comité a la mission, particulièrement importante pour l'avenir ćconomique du pays, détudier les opérations à entreplendre en rue de l'aménagemént de l'énergie hydraulique sur loute l'élendue du lesritoire ; à cet eflet, de procéder ì linventaire des principales chutes disponibles, de rechercher les moyens de les mettre en valeur et d'en réaliser l'emploi au micux des intérêts industriels ou agricoles.

S'appliquant à la France entièrc, ce problème, et notamment l'enquêle qu'il comporte, est extrèmement vaste. Ausí a-l-il paru nécessaire de subdiviser le comité en quatre souscomilés auxquels ont été respectivenent attribuées comme champ d'action les quatre grandes régions pourvues de forces'hydrauliques : Alpes, Pynénées, centre et Est.

D'après un programmé tracé par le comité, les sous-comités ont entrepris l'étude des multiples questions d'ordre tcchnique, économique et financier que comporte le sujet; ils se sont mis aclivement à l'œeuvre, et le comité qui doil contralisel lours travaux pourra ètre en mesure d'apporter diuns un très bref délai un certain nombre de résultats.

\section{d) Jistributions d'éneprgie électrique.}

Distributions d'énergie électrique. - le comité, dont le rôle consiste à étudier les mesures propres à favoriser l'utilisation de l'ćnergie électrique, a d'abord entrepris l'audition de-principales sociétés distributrices d'úlectricité ; elle en a enlendu plus de trente; il lua reste à en convoquer une yuinzaine. Les desiderata qui seront retenus pourront serviz de base à l'adoplion de nouvelle: dispositions destinées à amé. liorer, s'il y a lisu, la législation actuelle, et à favoriser ip développement des distributions.

En second lieu, le comité s'occupe de dresser une cart' gúnérale des distributions existantes, notamment des lignes do transport à haute tension. A l'aide de cette carte, on ponrua constater les lacunes existantes of aviser aux moyens à employer pour le; remplir. Le travail que le comité sé propose d'établir, comprendra : $I^{\circ}$ un tracé des nouvelles lignes à construirc ; $2^{\circ}$ leur situation, el $3^{\circ}$ leur classement par ordre d'urgenec. Ia production de ces documents exigera un certain délai que le comité s'efforcera d'abréger.

En altendant, il éludicra les disposilions faite- à l'enquête et examinera les conclusions à en tirer au point de vue du développement des distributions. 


\section{e) Ports maritimes.}

Nous ne publions pas le développement de ce chapitre.

\section{f) Chemins de fer.}

Amélioration des relations par voie ferrée entre la Suisse ct les ports de l'Ucéan. - Il a été rendu compte au second paragraphe de ce rapporl du résultat des lravaux du comnité chargé de l'ćlude de celle question.

Tunnea sous la Manche. -- Dans sa première séance, le comlé a entendu un exposé général de la question et a arrêté le programme de ses lravaux qu'il a décidé de faire porter sur les divers aspects, légal, technique, économique et linancier de l'alfaire.

Il a recomnu nécessaire de se préoccuper en premier lieu de saroir si la concession accordée par la loi du 2 aoùt $187^{5}$ ist encore valable, et d'examiner les que thons d'ordre international que soulève une concession de ce genre.

Les documonls à ce sujet se trouvent aujourd'hui réuns th te comité en délibérera dans une prochaine séance.

Los démarches auxquelles s'est livré le rapporteur du comité l'ont allené à apprendre que, du còté brilannique, nı le gouvernement, ni les compagnies intéressées ne comptent reprendre l'étude du tunnel sous la Manche tant que n'aura pas été résolue la questron, actuellement pendante, du futur régime des chemins de fer ; on considérerait en Angleterre que le régime actuel ne saurail subsisler el devrait farre place, soit à la nationalisation des réseaux soit à un système plus ou moins analogue au système français et de pourvoir à leur entretien ultérieur ;

$3^{\circ}$ Etudier toutes les questions de malériel intéressant l'amélioration de l'exploitation : freinage, outillage, ateliers, "agons métalliques, wagons frigorifiques, etc...

Il a été rendu compte au paragraphe 2 du présent rapport du résultat de leurs études sur les deux premières questions.

Leur travail se poursuit sur la troisième.

Matériel fixe et matériel roulant des réseaux d'intérêt local. - Ces deux comités ont, pour les voies ferrées d'intérêt lo(al, une mission analogue à celle des comités des grands rúscaux dont il vient d'être question.

Us ont produit, le ${ }^{\text {er }}$ décembre $19 \mathrm{I} 8$, un premier travail relatif aux mesures à prendre en vue d'approvisionner le ntatériel fixe et roulant nécessaire au nétablissement des voies lerrées d'intérêt local dans les régions dévastées, de réaliser i cette occasion la standarisation de ces deux matériels.

Etabli dans un délai extrêmement court après l'armistice, cr travail reposait sur des renseignements incomplets au sujet de l'état des lignes. Un supplément de délai leur ayant étć accordé, les comités ont repris leur étude sur de nouvelles bases.

Filectrification des réseaux d'intérêt général. - Dans sa première réunion, le comité a adopté un programme de travaux el nommé deux sous-commissions, l'une, dite administrative, et l'autre, dite technique.

La sous-commission administrative a provoqué et examiné les propositions d'électrification des trois réseaux : Midi, P. (). et P.-L..-M. ; ces propositions s'étendent au total sur 8. no kilomètres de lignes et comportent un programme de Iravaux (usines hydro-électriques, installations fixes de transformation et d'alimentation, matéricl tracteur) évalué à 2.400 millions.

Le rapport de la sous-commission sur ces propositions est 'n cours de rédaction et sera soumis à l'approbation du romité dans sa prochaine réunion.

Le rapporteur de la sous-comission technique s'occupe en ce moment d'exposer en un rapport documenté, l'expérience acluellement acquise, en Frauce el à lélranger, dans lélectriticalion des grandes lignes. Cic rappor $t$, destiné à faciliter la conparanson des divers systemes de traction actuellenent uhlisés, sera prochanement distribué à tous les membres du comité. Il servira de base à une discussion qui, après toutes vérificatıons exócutées par les missions jugées utiles par le comnté, porarra conduire à la détenmuation du choia du meilleur mode de traction électrique pour nos rénéaux d'intérêt général.

Cilassement dans le réseau dintérèt génćral de certaines lignes d'intérêt local. - Celte question se pose notamment it loccasion de leur reconstruction, pour certaines lignes détruites de, régions envahies, Le comlé envisage pour - quelques-unes d'entre elles l'opportunité de les transformer comme tracé, profil et largeur de loie, et en mème temps, de les incorporer dans le réseau d'inlérèt général. Les ingénieurs en chef du contròle ont été invités ì présenter leur. observations, le comité les attend pour formuler ses conclusions.

\section{Chemins de fer d'intérél général. - Lignes nouvelles.}

Le comité a été saisi đ'un état, préparé par l'adımmstration, des lignes ferrées déjà concédiés à hlle délinitif ou à titre íventuel, et de celles dont l'exécution pourrait ètre envisagée.

Les travaux préparatoires d'examen de cet état par le rapporteur sont en cours.

\section{g) Section des études générales.}

Enlin, la section des études générales a entrepris la préparation de mémoires sur les questions susvantes:

Utilisalion par la France du port d'Anvers. Transil international. Régime de tarification et dexploitation des voies de transoprt. Taxes perçues dans les ports. Droils de quai. Surtaxe d'entrepòt. Relations des transports par eau el par chemins de fer. Tarifs mixles.

Le champ d'action de ce service est destiné à s'étendre notablement lorsque sera achevée son organisation encore incomplète.

Telles sont, bric̀vement analysées, les principales questions - pour lesquelle- le Conseil supérieur des travaux publies a doriné à mon adminıstration sa pı́ccieuse collaboration. Avec une compétence, une activité et un xèle auxquels j'ai le devoir de rendre hommage ici. J'ai la pleine confiance que les solulions qu il prépare seront toujours prises, pour ce qui dépendra de lui, lorsque le moment sera venu de les réaliser.

\section{Note sur le Calcul de l'Espacement des Piliers} SUPPORTANT UNE CONDIITE SOUS PRESSION

La formule générale pour lo calcul d'une poulre soumise un effort de flexion est :

$$
\sigma=\frac{\mathrm{M}}{\mathrm{R}}
$$

dans laquell" f cal l'cfforl de traction ou de compression par unité de surface exercé sur la fibre siture à une distance $u$ de l'axe. noutre; M le moment fléchi-iant anqual la poutre est soumise ; R le module de résistance qui est égal à $\frac{1}{u}$-i T désigne le moment d'inertie de la section considérée. 
Dans le eas d'un tuyau, $M$ et $R$ ont los valeurs suivantes $M=\frac{P L^{2}}{10}$ (pontre semi-continue).

$\mathrm{P}=$ poids de la conduite pleine par unilé de longueur.

$\mathrm{I}_{2}=$ espacement entre les appuis.

$\mathrm{R}=\frac{\pi}{32} \frac{\mathrm{D}^{4}-d^{4}}{\mathrm{D}}$ ou approximativement $\cdot 0, \mathrm{D} \frac{\mathrm{D}^{k}-d^{k}}{\mathrm{D}}$

$d=$ diamètre intérieur du tıyau.

$D=$ diamòtre extérieur.
Il faut avoir soin, naturellement, d'employer les mêmes unités de poids et de longueur pour les différents termes de la formule (kilogrammes et centimètres, ou tonnes et mètres).

Dans le cas d'une conduite en pente, la longueur I, ealculéo au moyen de la formule précédente, représente la projer. tion horizontale de la distance entre les deux points d'appui

Pour déterminer la valeur admissible pour $z$, il y a lien Ar remarquer ce qui suit :

La pression de l'ean produit dans nmo conduite des efforts longitudinauy et transversaux.

Les efforts longitudinaux, c'est-à-dire reux qui tendent à faire rompre la conduite -uivant une directrice, sont moitié moundres que les efforts transversaux, qui tendent à faire ronıpre la conduite suivant une génératrice

En chaque point, les deux espèces d'clforls existent simullanément, mais on se confente de calculer les conduites en pression on tenanl compte du soul effort transloisal qui esl le plus grand.

Si la conduile repose sur des piliers, la llexion due à l'espacement des appuis créera des efforts l'ngıludinaux supplémentaires.

Ces effor'ts s'ajoutent ou se retranchent à ceux produits par la pression inférieure. Au milieu de la portée entre appuis, la flexion du tuyau provoquera une compression dans la fibre supérieure et une traction dans la libre inlérieure.

L'effort supplémentaire dû à la flexion du tuyau soulagera donc la fibre supérieure, mais augmentera l'effort de traction dû à la pression de l'eau dans la fibre inférieure.

En tenant compte du fait que des deux efforts dus à la pression de l'eau,c'est au plus faible, snit à l'cffort longitudinal, que vient -ajculor l'effort longitudinal supplémenlaire produil par la flexion du luyau, on en déduit que la valeur de $\sigma$ pourra atteindre la moitié du taux du travail pour lequel la conduite aura été calculée.

Lc diagramme qui suit a été établi pour des conduites métalliques en tôle rivée de 3.00 à $2000^{\mathrm{m} / \mathrm{m}}$ de diamèlre intérieur, calculées pour que le travail do la tôle, ne dépasse pas $6 \mathrm{kilogs}$ par $\mathrm{mm}^{2}$.

L'espacement des piliers a été déterminé au moyen de la formulc précédente dans laquelle on a admis poùr la valeur de o la moitié du taux du Iravail pour lequel la conduite a été calculćc, soit $\sigma=3 \mathrm{kgs}$ par $\mathrm{mmn}^{2}$.
En remplaçant M el $R$ par les valeurs ci-dessus dans la formule générale, on en déduit la valeur do l'espacement I, savoir :

$$
L=\sqrt{\frac{\sigma}{\mathrm{P}} \frac{\mathrm{D}^{4}-d^{4}}{\mathrm{D}}}
$$

Pour obtenir l'espacement admissible des pilicrs d'une conduite travaillant à $8 \mathrm{kgs}$ par $\mathrm{mm}^{2}$, il suffira de multiplier les distances. $L$ données par le diagrammo par $\frac{\sqrt{8}}{\sqrt{6}}=\cdot \mathrm{I}$, 5 .

Prilly, Décembre rgr8.

H. Cimenaud, Ingénient. 